\title{
Multi-hadron systems in lattice QCD
}

\author{
William Detmold [NPLQCD Collaboration] * \\ Department of Physics, College of William and Mary, Williamsburg, VA 23185, USA \\ Thomas Jefferson National Accelerator Facility, 12000 Jefferson Ave, Newport News, VA 23606, \\ USA. \\ E-mail: wdetmoldejlab.org
}

Lattice QCD is currently entering the stage when it can usefully be applied to systems of multiple hadrons. I briefly review the status of recent calculations of scattering parameters in the two hadron sector and discuss recent calculations of systems composed of many mesons or baryons. In the mesonic case, the NPLQCD collaboration has continued its study of systems of up to twelve pions or kaons and have computed the effect of such a hadronic medium on the static quark potential. High statistics calculations on anisotropic lattices have allowed for precision extraction of the energies and scattering phase shifts of various two baryon systems and, for the first time, the energies of certain three baryon systems have been computed.

The XXVII International Symposium on Lattice Field Theory - LAT2009

July 26-31 2009

Peking University, Beijing, China

\footnotetext{
*I am greatful to my colleagues in the NPLQCD collaboration for enjoyable and productive collaboration.
} 


\section{Introduction}

Lattice QCD has had major impact in many aspects of particle physics phenomenology and in describing the spectra and structure of single hadrons. Numerical algorithms and computational resources have reached a stage where it is now worthwhile to consider more complicated hadronic observables such as those in the baryon number, $B>1$ sector - the realm of nuclear physics. Here, there are many observables that are phenomenologically important to nuclear structure and interactions and to nuclear astrophysics about which very little (or nothing) is known experimentally or theoretically. Two important examples are the hyperon-nucleon interactions that enter in the equation of state governing the evolution of a supernova and the three-body interactions necessary to describe the structure of nuclei. Systems containing $n \geq 2$ mesons are also of interest in a number of areas from relativistic heavy ion collisions to neutron stars. These systems present a significant opportunity for contributions from lattice QCD.

For systems comprised of two hadrons, it has been known for many years that, below inelastic thresholds, the dependence of the eigen-energies on the spatial lattice volume is directly related to the corresponding two-particle phase shift at that energy. This has been known in quantum mechanical systems for many years [1] but was proven in the field theory context by Lüscher [2] and only relies on the lattice size, $L$, being large compared to the range of the interaction, $R \sim m_{\pi}^{-1}$, such that the two hadron wave-function attains its (unique) asymptotic form. In recent years, this method has been utilised to study interactions between light mesons, between mesons and baryons, between baryons themselves, and between baryons and mesons and various hadrons containing heavy quarks. In Section 2, we review the methodology and recent results.

Recently, the first studies of systems of more than two hadrons have been made by the NPLQCD collaboration $[3,4,5,6]$. Mesonic systems involving up to twelve pions or kaons have been constructed numerically and the two and three body interactions in these systems and also dependence of the corresponding isospin or hypercharge chemical potentials on hadron density were extracted. The effects of the hadronic medium formed in these calculations was also investigated. For baryon systems, the baryon number $B=3$ sector has recently been studied for the first time in lattice QCD and important observations regarding statistical noise have given hope to the extension of these calculations to larger $B$. The results of these studies, for both mesons and baryons, are summarised herein.

\section{Two-particle systems}

The last few years have seen significant developments in the study of hadron-hadron interactions in lattice QCD. Here we review how information about hadronic interactions is obtained from Euclidean correlation functions computed in lattice QCD and survey the recent results that have been presented.

As first shown by Lüscher in the context of a quantum field theory [2], the energy of two particles in a finite spatial volume depends, in a calculable way, upon their elastic scattering amplitude and their masses for energies below the inelastic threshold. To demonstrate this method, we consider a correlation function with the quantum numbers of a $\pi^{+} \pi^{+}$(isospin $I=2$ ) system in which 
the particles are moving with relative momentum $\mathbf{p}$

$$
C_{\pi^{+} \pi^{+}}(p, t)=\sum_{|\mathbf{p}|=p} \sum_{\mathbf{x}, \mathbf{y}} e^{i \mathbf{p} \cdot(\mathbf{x}-\mathbf{y})}\left\langle\pi^{-}(t, \mathbf{x}) \pi^{-}(t, \mathbf{y}) \pi^{+}(0, \mathbf{0}) \pi^{+}(0, \mathbf{0})\right\rangle
$$

where $\pi^{+}(\mathbf{x}, t)=\bar{u}(\mathbf{x}, t) \gamma_{5} d(\mathbf{x}, t)$. Typically, the energy difference between the interacting and noninteracting two-meson states is a small fraction of the total energy, which is dominated by the masses of the mesons. In order to extract this energy difference we use the single pion two-point correlation function

$$
C_{\pi}(t)=\sum_{\mathbf{x}}\left\langle\pi^{-}(t, \mathbf{x}) \pi^{+}(0, \mathbf{0})\right\rangle
$$

to construct the ratio of correlation functions

$$
\frac{C_{\pi^{+} \pi^{+}}(p, t)}{C_{\pi}(t) C_{\pi}(t)} \rightarrow \sum_{n=0}^{\infty} Z_{n} e^{-\delta E_{n} t}
$$

where the last relation is valid for time-slices $t$ physically separated from $t=0$. The energy shifts, $\delta E_{n}$, that appear in this expression are the deviation of the two-particle energy from the sum of the rest masses of the particles and are related to the interacting center-of-mass momentum $p_{n}$ by

$$
\delta E_{n} \equiv E_{n}-2 m_{\pi}=2 \sqrt{p_{n}^{2}+m_{\pi}^{2}}-2 m_{\pi} .
$$

To obtain $p \cot \delta(p)$, where $\delta(p)$ is the phase shift, the square of the center-of-mass momentum, $p$, is determined from fits of the appropriate energy shifts in Eq. (2.3) and inserted into

$$
p \cot \delta(p)=\frac{1}{\pi L} \mathbf{S}\left(\left(\frac{p L}{2 \pi}\right)^{2}\right),
$$

which is valid below the inelastic threshold. The regulated three-dimensional sum is [7]

$$
\mathbf{S}(x) \equiv \lim _{\Lambda \rightarrow \infty} \sum_{\mathbf{j}}^{|\mathbf{j}|<\Lambda} \frac{1}{|\mathbf{j}|^{2}-x}-4 \pi \Lambda,
$$

where the summation is over all triplets of integers $\mathbf{j}$ such that $|\mathbf{j}|<\Lambda$ and the limit $\Lambda \rightarrow \infty$ is implicit. Therefore, by measuring the energy-shift, $\Delta E_{n}$, of the two particles in the finite lattice volume, the scattering phase-shift is determined at $\Delta E_{n}$. In the absence of interactions between the particles, $|p \cot \delta|=\infty$, and the energy eigenstates in the finite volume occur at the expected momenta $\mathbf{p}=2 \pi \mathbf{j} / L$. This expression is valid for any (even infinite) scattering lengths [7]. The only restriction is that the lattice volume be much larger than the range of the interaction between the hadrons, which is typically set by the mass of the pion.

An alternative method $[8,9]$ considers the two particle wave function at separations outside the range of the interaction where asymptotic scattering solutions are valid. In this region, direct matching to the expected asymptotic behaviour of scattering solutions is possible and the phase shift can be extracted at the energy of the system under consideration. ${ }^{1}$ The information obtained

\footnotetext{
${ }^{1}$ It is possible to use the wave-functions measured in this way to define a two-particle potential (see Ref. [10] for a review). However, it is important to stress $[11,12,13]$ that this potential is dependent on the choice of interpolating operators used in the construction of the wave function (unlike the phase shift). The potential extracted in this manner is an energy-dependent object formally containing the same information as the phase shift at the particular energy as can be extracted from the Lüscher method. The reader is referred to the proceedings of N. Ishii at this conference [14] for further discussion.
} 
from this method is formally equivalent to that which can be extracted from the eigen-energies. Nevertheless, the systematics of the two methods differ significantly and it is not yet clear which approach will ultimately extract the most precise physical results for a given computational effort.

\subsection{Meson-meson interactions}

For the case of interactions between pseudoscalar mesons, a number of groups have recently reported results with precision that is better than that of experimental determinations. This is particular to the case of interactions in channels without annihilation contributions, namely, $I=2$ $\pi \pi, I=3 / 2 K \pi$ and $I=1 K K$ systems. There were a number of contributions to this conference that highlighted this $[15,16,17]$. In addition, some progress has been made regarding the numerically more challenging channels, such as $I=0,1 \pi \pi$ interactions [16, 18, 19] (see also Ref. [20]).

\subsection{Meson-baryon systems}

In the meson-baryon sector, there has been recent progress from the NPLQCD collaboration [21] using domain wall fermions on MILC gauge configurations. The five possible meson-baryon channels with no annihilation contributions $\left(\pi^{+} \Sigma^{+}, \pi^{+} \Xi^{0}, K^{+} p, K^{+} n\right.$ and $\left.\bar{K}^{0} \Xi^{0}\right)$ were studied and phase shifts were extracted at multiple quark masses, and at two lattice spacings. Both 2and 3-flavour chiral perturbation theory analyses were used to extrapolate to the physical point. For the interactions involving kaons, the convergence of the chiral expansion at the quark masses used in the lattice calculations was found to be poor, however consistent results were obtained for the channels involving pions and the scattering lengths in these channels were determined to be $a_{\pi^{+} \Sigma^{+}}=-0.197(17)$ fm and $a_{\pi^{+} \Xi^{0}}=-0.098(17)$ fm with the comprehensive error encompassing statistical and systematic uncertainties. These results deviate from the current algebra (leading order chiral perturbation theory) predictions at the one- and two-sigma levels, respectively.

The first measurement of a meson-baryon interaction involving disconnected contributions was also recently presented in Ref. [22] where the $s$-wave $I=\frac{1}{2} \pi N$ phase shift was extracted at one particular energy and at a particular pion mass, $m_{\pi}=390 \mathrm{MeV}$. These calculations are encouraging as it is the channels involving disconnected contractions that are physically relevant in nuclear astrophysics phenomenology.

\subsection{Baryon-baryon systems}

A number of results have been reported recently for baryon-baryon interactions from the HALQCD and NPLQCD collaborations. Significant new results for the various hyperon-nucleon interactions that are important in nuclear astrophysics have recently been presented [23, 24, 25, 26, 27]. Fig. 1 summarises global results for the various hyperon-nucleon channels, however, care should be taken in comparing the various results for a given channel as the displayed phase shifts are evaluated at significantly different scattering momenta.

\subsection{Systems with heavy quarks}

There have also been recent calculations of interactions between various hadrons containing charm quarks and between heavy and light hadrons. Channels studied include the phenomenologically relevant $J / \Psi-N, D-D^{*}$ and $K-D$ channels that may answer questions such as whether 

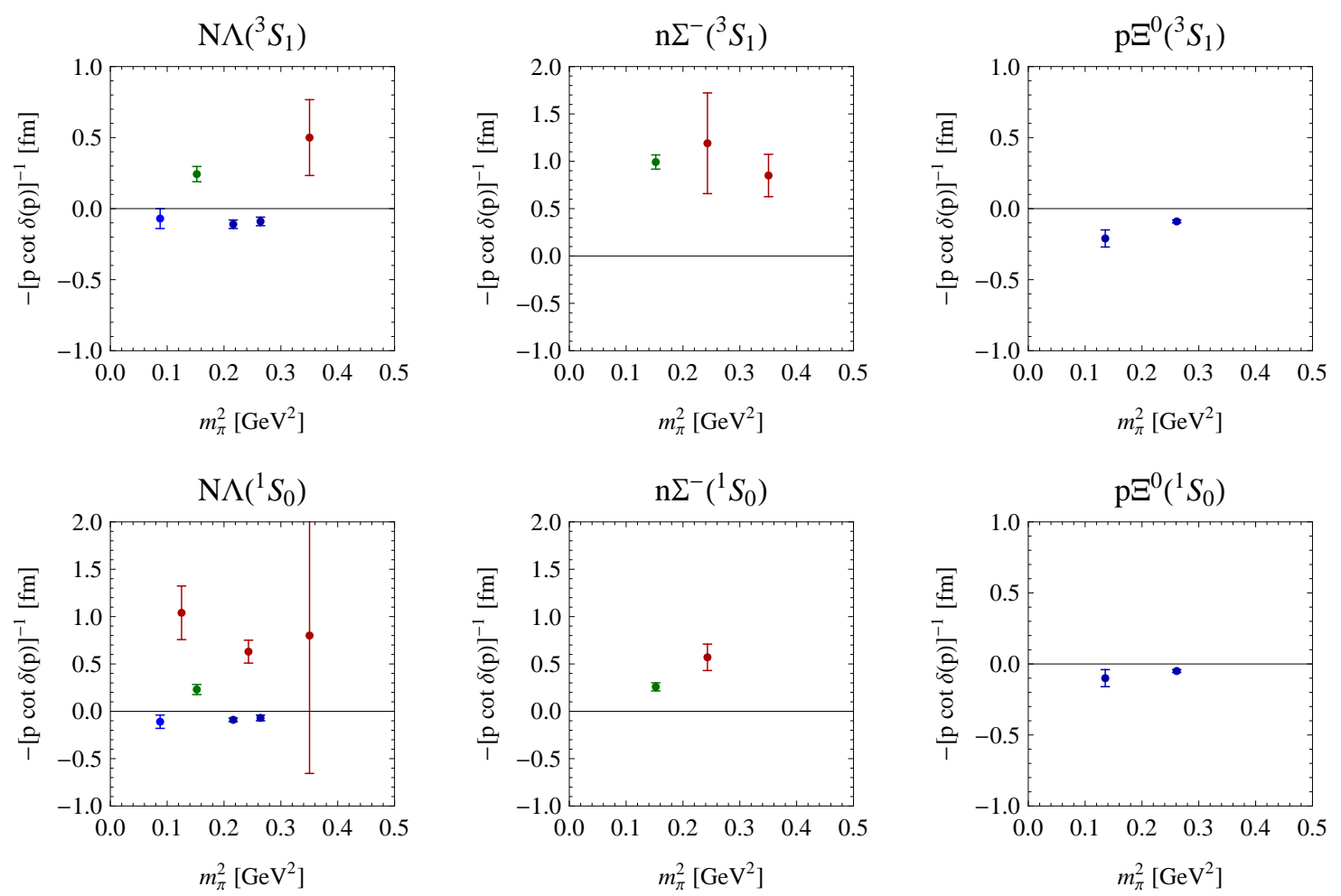

Figure 1: Summary of the hyperon-nucleon phase shift determinations from recent calculations, . Results are displayed on a single plot for each channel for concision, however direct comparison of the various calculations is not yet possible as the phase shifts are evaluated at different momenta. The various symbols correspond to result from Refs. [24] (green), [25] (red), [26] (dark blue; $p \Xi^{0}$ ) and [27] (dark and light blue $N \Lambda)$.

charmonium can be bound in nuclei and what is the nature of the various charmonium-like XYZ mesons? These results were discussed in contribution of L. Liu [28].

\section{Multi-meson systems}

It has been known for many years how to exploit the volume dependence of the eigen-energies of two hadron systems to extract infinite volume scattering phase shifts [2] provided that the effective range of the interaction, $r$ is small compared to the box size $L$ (since $r \sim m_{\pi}^{-1}$ for most interactions, this constraint is $m_{\pi} L \gg 1$ ). In recent works, this has been extended to systems involving $n>2$ bosons [29, 30, 31] and $n=3$ fermions [32] in the case when the relevant scattering length, $a$, is also small compared to the box size. The resulting shift in energy of $n$ bosons of mass $M$ due to their interactions is

$$
\begin{aligned}
\Delta E_{n}=\frac{4 \pi \bar{a}^{n}}{M L^{3}} C_{2}\{1 & -\left(\frac{\bar{a}}{\pi L}\right) \mathscr{I}+\left(\frac{\bar{a}}{\pi L}\right)^{2}\left[\mathscr{I}^{2}+(2 n-5) \mathscr{J}\right] \\
& -\left(\frac{\bar{a}}{\pi L}\right)^{3}\left[\mathscr{I}^{3}+(2 n-7) \mathscr{I} \mathscr{J}+\left(5 n^{2}-41 n+63\right) \mathscr{K}\right]
\end{aligned}
$$




$$
\begin{gathered}
+\left(\frac{\bar{a}}{\pi L}\right)^{4}\left[\mathscr{I}^{4}-6 \mathscr{I}^{2} \mathscr{J}+\left(4+n-n^{2}\right) \mathscr{J}^{2}+4\left(27-15 n+n^{2}\right) \mathscr{I} \mathscr{K}\right. \\
\left.\left.+\left(14 n^{3}-227 n^{2}+919 n-1043\right) \mathscr{L}\right]\right\} \\
+{ }^{n} C_{3}\left[\frac{192 \bar{a}^{5}}{M \pi^{3} L^{7}}\left(\mathscr{T}_{0}+\mathscr{T}_{1} n\right)+\frac{6 \pi \bar{a}^{3}}{M^{3} L^{7}}(n+3) \mathscr{I}\right] \\
+{ }^{n} C_{3} \frac{1}{L^{6}} \overline{\bar{\eta}}_{3}^{L}+\mathscr{O}\left(L^{-8}\right)
\end{gathered}
$$

where the parameter $\bar{a}$ is related to the scattering length, $a$, and the effective range, $r$, by

$$
a=\bar{a}-\frac{2 \pi}{L^{3}} \bar{a}^{3} r\left(1-\left(\frac{\bar{a}}{\pi L}\right) \mathscr{I}\right) .
$$

The geometric constants that enter into eq. (3.1) are defined in Ref. [31] and ${ }^{n} C_{m}=n ! / m ! /(n-m) !$. The three-body contribution to the energy-shift given in eq. (3.1) is represented by the parameter $\overline{\bar{\eta}}_{3}^{L}$ (see Ref. [31]). The analogous results for mixed systems of two species of mesons have recently been derived to $\mathscr{O}\left(1 / L^{6}\right)$ accuracy in Ref. [33].

Lattice QCD measurements of these energy shifts allow one to extract the parameters $\bar{a}$ and $\overline{\bar{\eta}}_{3}^{L}$. To determine the energy shifts, we study the correlators (specifying to the multi-pion system)

$$
C_{n}(t) \propto\left\langle\left(\sum_{\mathbf{x}} \pi^{-}(\mathbf{x}, t)\right)^{n}\left(\pi^{+}(\mathbf{0}, 0)\right)^{n}\right\rangle .
$$

On a lattice of infinite temporal extent, ${ }^{2}$ the combination

$$
G_{n}(t) \equiv \frac{C_{n}(t)}{\left[C_{1}(t)\right]^{n}} \stackrel{t \rightarrow \infty}{\longrightarrow} \mathscr{B}_{0}^{(n)} e^{-\Delta E_{n} t}
$$

where $\Delta E_{n}$ is the ground state energy shift appearing in Eq. (3.1).

To compute the $(n !)^{2}$ Wick contractions in Eq. (3.3), we note that this correlation function can be written as

$$
C_{n}(t) \propto\left\langle(\bar{\eta} \Pi \eta)^{n}\right\rangle
$$

where

$$
\Pi=\sum_{\mathbf{x}} S(\mathbf{x}, t ; 0,0) S^{\dagger}(\mathbf{x}, t ; 0,0),
$$

and $S(\mathbf{x}, t ; 0,0)$ is a light-quark propagator. The object (block) $\Pi$ is a $12 \times 12$ (4-spin and 3 color) bosonic time-dependent matrix, and $\eta_{\alpha}$ is a twelve component Grassmann variable. The required $n$-meson contractions can be expressed as products of traces of the this matrix. As an example, the contractions for the $3-\pi^{+}$system are

$$
C_{3}(t) \propto \operatorname{tr}_{\mathrm{C}, \mathrm{S}}[\Pi]^{3}-3 \operatorname{tr}_{\mathrm{C}, \mathrm{S}}\left[\Pi^{2}\right] \operatorname{tr}_{\mathrm{C}, \mathrm{S}}[\Pi]+2 \operatorname{tr}_{\mathrm{C}, \mathrm{S}}\left[\Pi^{3}\right],
$$

where the traces, $\operatorname{tr}_{C, S}$, are over color and spin indices. Contractions for $n \leq 12$ mesons are given explicitly in Ref. [4].

\footnotetext{
${ }^{2}$ Effects of temporal (anti-)periodicity are discussed in Ref. [5].
} 


\subsection{Two- and three- body interactions}

The NPLQCD collaboration have computed the $n$ pion and kaon correlators in the previous section using domain wall fermion [34, 35] propagators on various ensembles of MILC 2+1 flavour rooted staggered gauge configurations [36] (quark masses, lattice spacings and volumes and further details are given in Refs. [3, 4, 5]). In order to correctly calculate these correlators for large $n$, very high numerical precision is necessary. By performing a correlated fit to the effective energy differences extracted from these measurements, we have determined the two- and three-body interactions. The two body interactions extracted from this analysis agree with those extracted from the two-body sector alone [37]. The resulting three body interactions are displayed in Fig. 2. The three pion interaction is found to be repulsive with a magnitude consistent with the expectation from naive dimensional analysis. In contrast, the three $K^{+}$interaction is consistent with zero within somewhat larger uncertainties.
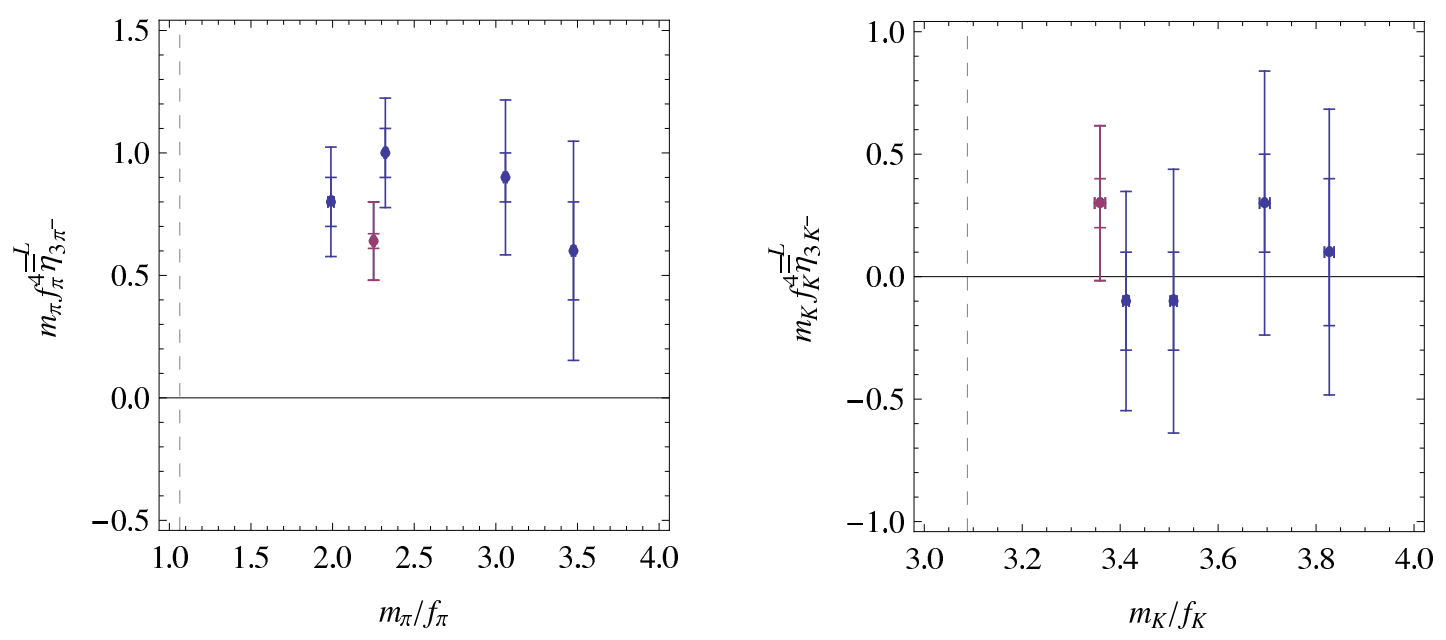

Figure 2: Three pion (left) and kaon (right) interactions determined from the MILC coarse (blue) and fine (magenta) lattices plotted as a function of the dimensionless ratios $m_{\pi} / f_{\pi}$ and $m_{K} / f_{K}$ respectively.

\subsection{Pion and kaon condensation}

The ground state of the $n$ meson systems that are being studied is a Bose-Einstein condensate of fixed $z$ component of isospin (and strangeness in the case of kaons). It is of great interest to investigate the properties of such systems. Theoretical efforts have used leading order chiral perturbation theory to investigate the phase diagram at low chemical potential [38] and it is important to assess the extent to which these results agree with QCD. Our numerical calculations allow us to probe the dependence of the energy on the pion (kaon) density, and thereby extract the chemical potential via a finite difference. The results using the coarse MILC lattice are shown for kaon systems in Fig. 3. Also shown is the prediction from tree-level chiral perturbation theory, with which we find surprisingly good agreement. This is encouraging for studies of kaon condensation in neutron stars where, typically, tree level chiral perturbation theory interactions are assumed amongst kaons and between kaons and baryons [39]. 

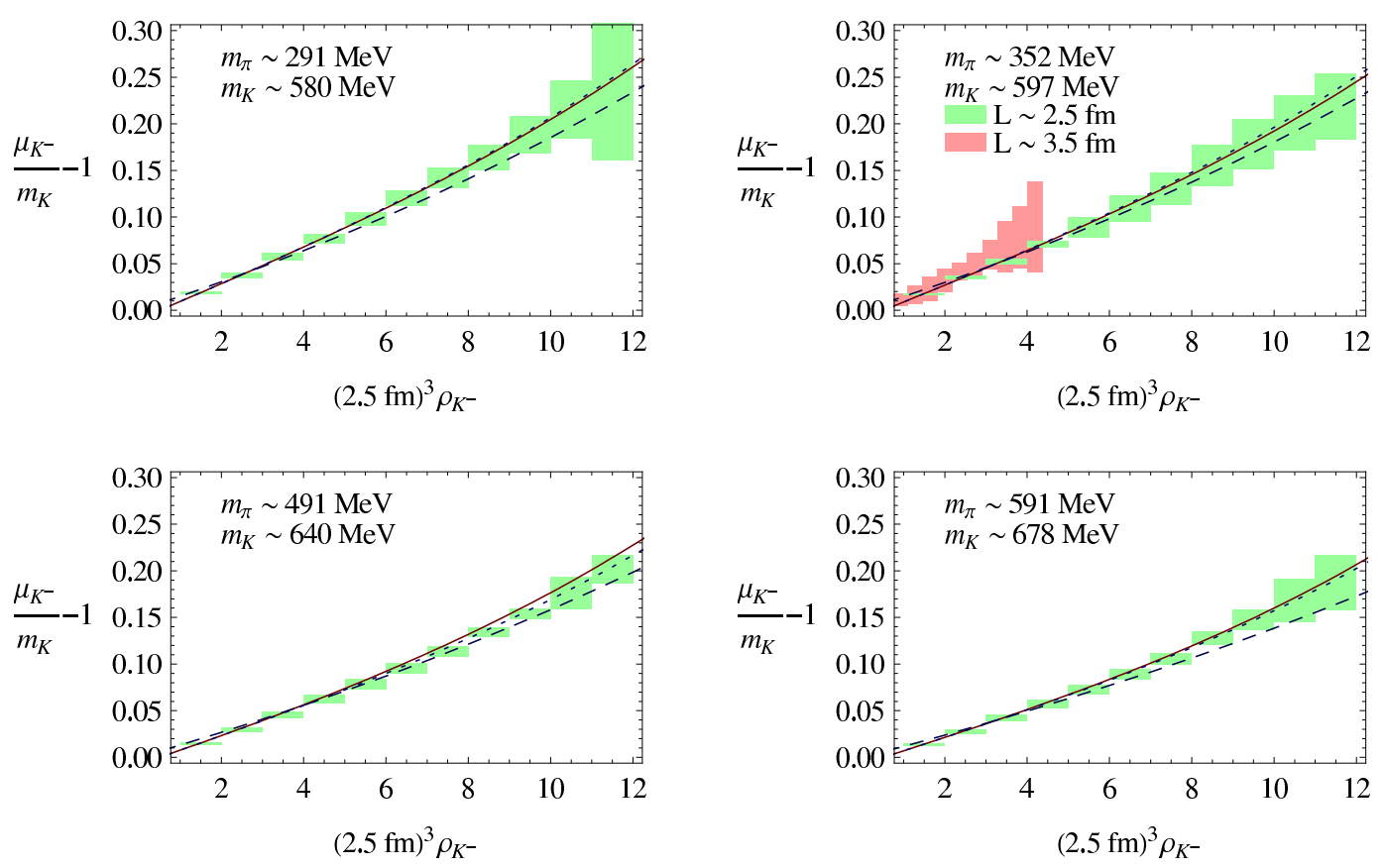

Figure 3: Dependence of the strangeness chemical potential on the kaon density, calculated on the coarse MILC ensembles. The curves correspond to the predictions of tree level chiral perturbation theory (dashed) [38], the energy shift of Eq. (2.1) (solid) and with the three-body interaction removed (dotted).

\subsection{Hadronic medium effects}

The hadronic medium formed in these many-meson calculations is naturally expected to influence the properties of other hadrons that interact with it. A phenomenologically relevant example is the passage of a $J / \Psi$ meson through the medium as $J / \Psi$ suppression is taken to be a key signal for the formation of a quark-gluon plasma [40]. In Ref. [41], a simplified version of this was considered, and the screening of the static quark potential by a condensed pion gas was computed. In order to extract the shift in the static potential caused by the presence of the medium, the following ratio of correlation functions was studied

$$
G_{n, W}\left(R, t_{\pi}, t_{w}, t\right)=\frac{C_{n, W}\left(R, t_{\pi}, t_{w}, t\right)}{C_{n}\left(t_{\pi}, t\right) C_{W}\left(R, t_{w}, t\right)},
$$

where $C_{n}$ is defined in Eq. (3.3) and

$$
\begin{aligned}
C_{W}\left(R, t_{w}, t\right) & =\left\langle 0\left|\sum_{\mathbf{y},|\mathbf{r}|=R} \mathscr{W}\left(\mathbf{y}+\mathbf{r}, t ; \mathbf{y}, t_{w}\right)\right| 0\right\rangle, \\
C_{n, W}\left(R, t_{\pi}, t_{w}, t\right) & =\left\langle 0\left|\left[\sum_{\mathbf{x}} \pi^{-}(\mathbf{x}, t) \pi^{+}\left(0, t_{\pi}\right)\right]^{n} \sum_{\mathbf{y},|\mathbf{r}|=R} \mathscr{W}\left(\mathbf{y}+\mathbf{r}, t ; \mathbf{y}, t_{w}\right)\right| 0\right\rangle,
\end{aligned}
$$

and $\mathscr{W}\left(\mathbf{y}, t_{0} ; \mathbf{y}+\mathbf{r}, t\right)$ is the Wilson-loop operator formed from products of gauge-links joining the vertices at $\left(\mathbf{y}, t_{0}\right),\left(\mathbf{y}+\mathbf{r}, t_{0}\right),(\mathbf{y}+\mathbf{r}, t)$ and $(\mathbf{y}, t)$. At large Euclidean time, this ratio falls exponentially with a scale $\delta V(R, n)$ that is the shift in static potential for separation $R=|\mathbf{r}|$ and isospin density $\rho=\rho_{0} n$ (where $\rho_{0}=1 / V \sim 0.064 \mathrm{fm}^{-3}$ ). Further details are discussed in Ref. [41]. 


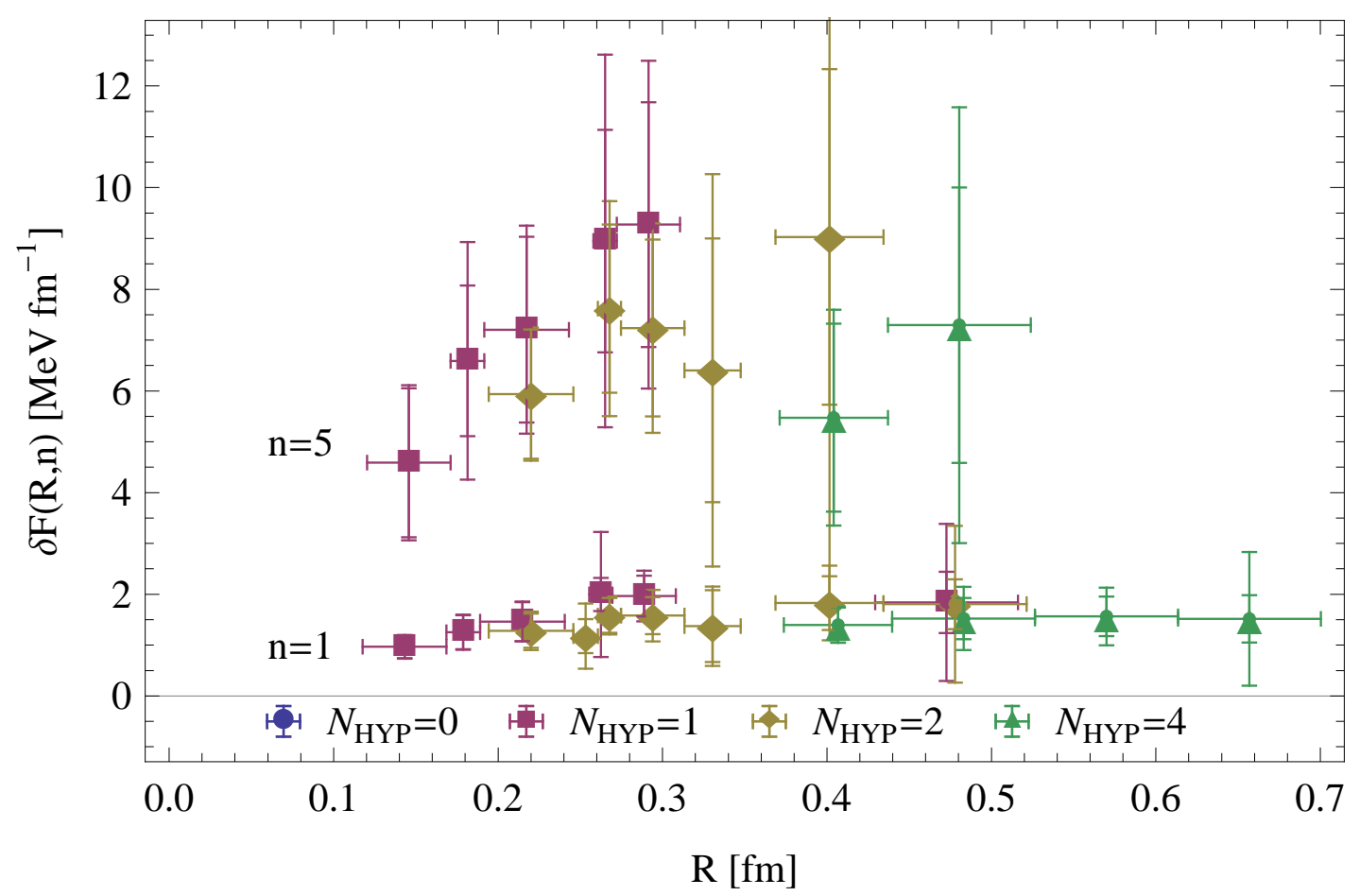

Figure 4: The in-medium contribution to the radial $Q \bar{Q}$ force, $\delta F(R, n)$, at a pion number density of $\rho_{0}$ and $5 \rho_{0}$.The inner uncertainty associated with each point is statistical, while the outer is the statistical and systematic uncertainties combined in quadrature.

As expected from the weak nature of pion interactions with isoscalar objects, the overall screening effect is small. In the region where the force between the static quark and anti-quark is constant, $F \sim 1 \mathrm{GeVfm}^{-1}$, the shift is $\mathscr{O}\left(\mathrm{MeVfm}^{-1}\right)$. The shift in the force was found to vary linearly with the isospin density of the hadronic system as shown in Fig. 4. This is consistent with a simple interpretation in terms of a dielectric in the volume of the colour flux tube between the quark-anti-quark pair. Further calculations extending this study to dynamical charm quarks are underway. To directly connect with phenomenology, the more difficult problem of finite baryon density must be confronted.

\section{Multi-baryon systems}

In Ref. [6], the first lattice QCD calculation of three baryon systems were presented based on extremely high statistics measurements on anisotropic lattice configurations. These calculations used anisotropic clover lattices generated by the Hadron Spectrum Collaboration [42, 43] and focused primarily on a strangeness $S=4, I_{z}=1 / 2$ system " $\Xi^{0} \Xi^{0} n^{n}$ ". Preliminary results for the triton, pnn were also presented. The energies of the " $\Xi^{0} \Xi^{0} n$ " system was extracted with a relative precision that is within a factor of two of the extraction of single baryon energies, $E_{\Xi^{0} \Xi^{0} n}=3877.9 \pm 6.9 \pm 9.2 \pm 3.3 \mathrm{MeV}$ corresponding to an energy-shift due to interactions of $\delta E_{\Xi^{0} \Xi^{0} n}=E_{\Xi^{0} \Xi^{0} n}-2 M_{\Xi^{0}}-M_{n}=4.6 \pm 5.0 \pm 7.9 \pm 4.2 \mathrm{MeV} .^{3}$

\footnotetext{
${ }^{3}$ While the precision of these calculations was not sufficient to resolve a nonzero energy shift in this system at
} 
At present, unlike the situation in multi-meson systems discussed above [3, 4, 5], the analytical tools are not in place to use the above energy shift and those of the associated two baryon systems to extract the parameters describing the relevant two- and three-body interactions. While the volume dependence of the simplest three-fermion systems has been studied in Ref. [32], the mixing we expect between four closely spaced states (naively, systems comprised of $\Xi^{0} \Xi^{0} n, \Xi^{0} \Lambda \Lambda, \Xi^{-} \Sigma^{+} \Lambda$, $\Xi^{0} \Sigma^{+} \Sigma^{-}$and their isospin partners are close in energy) complicates the situation.

An important result of this study was the realisation that the exponential growth of statistical noise expected from simple arguments first presented by Lepage [44] can be suppressed. The generalised effective energy for the $\Xi^{0} \Xi^{0} n$ state is shown in Fig. 5. The salient point is that for a large range of time-slices, the uncertainties it the measured correlation function remain essentially constant. In this "golden window", fits can be performed to the correlator and the energies can be cleanly extracted. This region appears to shrink as baryon number, $B$, increases and it is instructive to delve into this behaviour in detail.

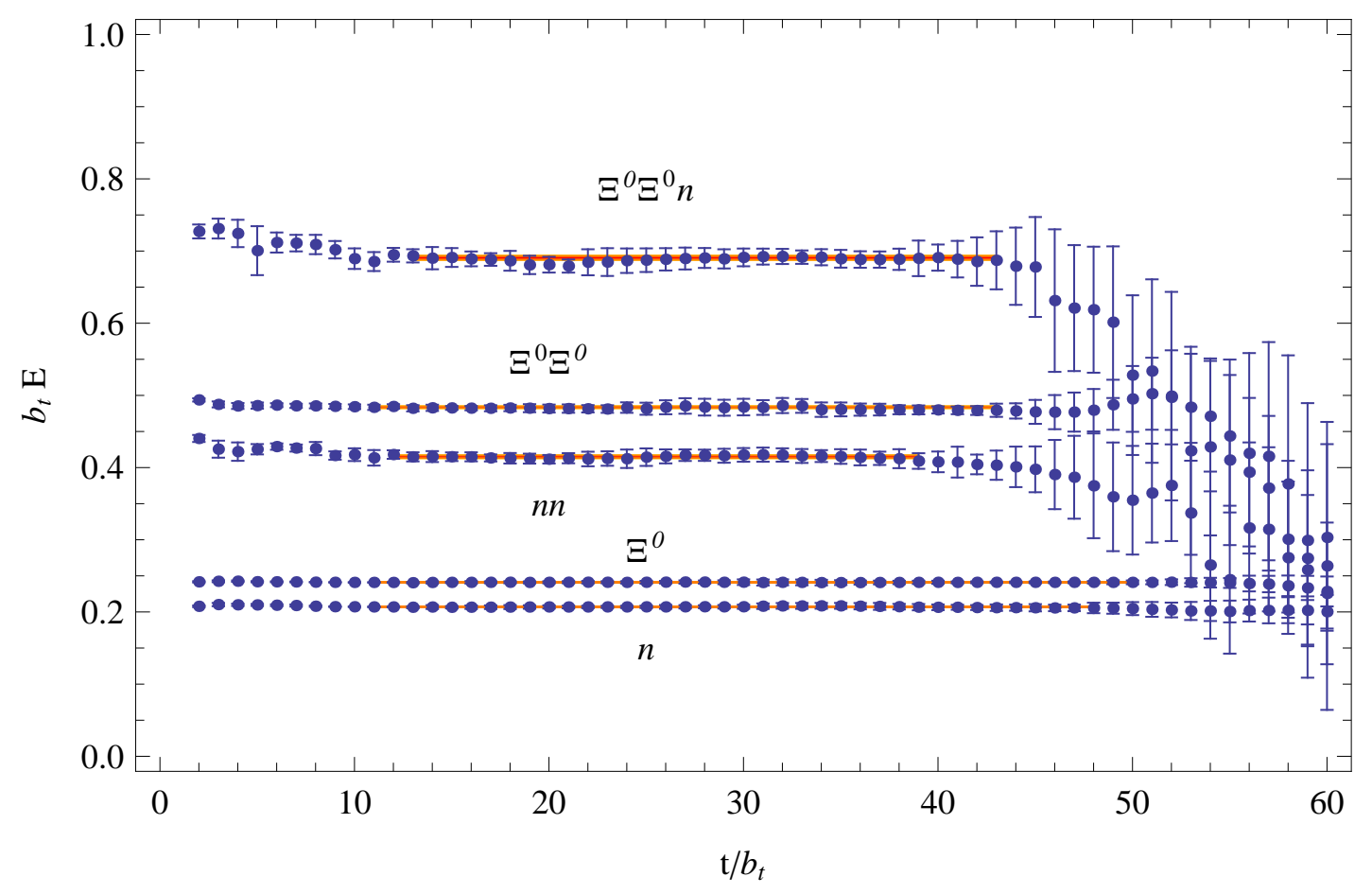

Figure 5: The effective energy plot for the one-, two- and three- body systems involving the $\Xi^{0}$ and $n$.

As argued by Lepage [44], correlation functions involving one or more baryons exhibit statistical noise that increases exponentially with Euclidean time. ${ }^{4}$ In the case of a single positive parity nucleon, the correlation function has the form

$$
\left\langle\theta_{N}(t)\right\rangle=\sum_{\mathbf{x}} \Gamma_{+}^{\beta \alpha}\left\langle N^{\alpha}(\mathbf{x}, t) \bar{N}^{\beta}(\mathbf{0}, 0)\right\rangle \rightarrow Z_{N} e^{-M_{N} t},
$$

$m_{\pi}=390 \mathrm{MeV}$, we note that it would be sufficient to resolve the typical nuclear binding energies of 7-8 MeV per nucleon.

${ }^{4}$ There is an connection between this issue and the sign problem at finite baryon density. 
where $N^{\alpha}(\mathbf{x}, t)$ is an interpolating field that has non-vanishing overlap with the nucleon, $\Gamma_{+}$is a positive energy projector, and the angle brackets indicate statistical averaging over measurements on an ensemble of configurations. The variance of this correlation function is given by

$$
\begin{aligned}
\mathrm{N} \sigma^{2} & \sim\left\langle\theta_{N}^{\dagger}(t) \theta_{N}(t)\right\rangle-\left\langle\theta_{N}(t)\right\rangle^{2} \\
& =\sum_{\mathbf{x}, \mathbf{y}} \Gamma_{+}^{\beta \alpha} \Gamma_{+}^{\gamma \delta}\left\langle N^{\alpha}(\mathbf{x}, t) \bar{N}^{\beta}(\mathbf{y}, t) N^{\gamma}(\mathbf{0}, 0) \bar{N}^{\delta}(\mathbf{0}, 0)\right\rangle-\left\langle\theta_{N}(t)\right\rangle^{2} \\
& \rightarrow Z_{N} e^{-2 M_{N} t}-Z_{N}^{2} e^{-2 M_{N} t}+Z_{3 \pi} e^{-3 m_{\pi} t}+\ldots \rightarrow Z_{3 \pi} e^{-3 m_{\pi} t}
\end{aligned}
$$

where all interaction energies have been neglected, and $\mathrm{N}$ is the number of (independent) measurements (distinct from the nucleon field operator $N$ ). Therefore, at large times, the noise-to-signal ratio behaves as

$$
\frac{\sigma}{\bar{x}}=\frac{\sigma(t)}{\langle\theta(t)\rangle} \sim \frac{1}{\sqrt{\mathrm{N}}} e^{\left(M_{N}-\frac{3}{2} m_{\pi}\right) t} .
$$

More generally, for a system of $A$ nucleons, the noise-to-signal ratio behaves as

$$
\frac{\sigma}{\bar{x}} \sim \frac{1}{\sqrt{\mathrm{N}}} e^{A\left(M_{N}-\frac{3}{2} m_{\pi}\right) t}
$$

at large times. The degradation of the signal-to-noise ratio on gauge-field configurations of finite temporal extent is exponentially more rapid than that given in Eq. (4.4) due to the presence of thermal states, as discussed in Ref. [22].

From the signals and variances that we have measured in the one-, two- and three-baryon sectors, it is clear that there is a suppression of the overlap onto the three-meson state from the $N \bar{N}$ source and sink (the variance correlation function of Eq. (4.2)), as encapsulated in the factor $Z_{3 \pi}$. If $Z_{3 \pi} \ll Z_{N \bar{N}}, Z_{N}^{2}$ there will be a number of time-slices, near the source of the correlation function, for which the noise-to-signal ratio behaves as

$$
\frac{\sigma}{\bar{x}} \sim \frac{1}{\sqrt{\mathrm{N}}}
$$

and does not depend exponentially upon time, or the differences of hadron masses. The correlation functions we have constructed lead to an implicit suppression of $Z_{3 \pi}$ compared to $Z_{N \bar{N}}$ and $Z_{N}^{2}$, due to the fact that the overlap onto the three-meson state, or any meson state, is strongly suppressed when the sinks $N^{\alpha}(\mathbf{x}, t)$ and $\bar{N}^{\beta}(\mathbf{y}, t)$ do not overlap within a volume approximately defined by the pion Compton wavelength. Therefore, summing independently over the volumes for $N^{\alpha}(\mathbf{x}, t)$ and $\bar{N}^{\beta}(\mathbf{y}, t)$ leads to a suppression factor that scales with the spatial lattice volume as $Z_{3 \pi} / Z_{N \bar{N}} \sim 1 / \Lambda^{3} L^{3}$. The pion mass dictates the weakest suppression, and hence we set $\Lambda=m_{\pi}$ for the estimates that follow (if the width, $w$, of the smearing of the source and sink is larger than the pion Compton wavelength, then $\Lambda \sim 1 / w$ ). Results consistent with this volume scaling have been found explicitly in calculations of single baryon energies using domain-wall fermions on MILC gauge configurations.

By generalizing this argument to systems composed of $A$ nucleons ${ }^{5}$ where each interpolating field is projected to zero momentum, the noise correlation function is expected to behave paramet-

\footnotetext{
${ }^{5}$ This simple argument holds for $A \leq 4$ nucleons and generalizes simply to $A \leq 16$ octet baryons. Further generalizations of this argument accounting for Fermi statistics are straightforward.
} 
rically as

$$
\begin{aligned}
\mathrm{N} \sigma^{2} \sim & \frac{(A !)^{2}}{\left(m_{\pi} L\right)^{3 A}} Z_{A} e^{-3 A m_{\pi} t}+\frac{((A-1) !)^{2}}{\left(m_{\pi} L\right)^{3(A-1)}} Z_{A-1} e^{-\left(2 M_{N}+3(A-1) m_{\pi}\right) t}+\ldots \\
& +\frac{A^{2}}{\left(m_{\pi} L\right)^{3}} Z_{1} e^{-\left(2(A-1) M_{N}+3 m_{\pi}\right) t}+Z_{0} e^{-2 A M_{N} t}+\ldots
\end{aligned}
$$

where we have made explicit the parametric dependence of the overlap factors on the baryon number and spatial volume. The dependence on $A$ arises from the number of ways that $N$ and $\bar{N}$ sink operators can overlap to form one or more three-pion contributions to the correlation function. Provided that the spatial volume is large compared to the Compton wavelength of the pion, $m_{\pi} L \gg 1$, there will be a range of time-slices in which only the last two terms in Eq. (4.6) are important. In this region, the signal-to-noise ratio in the multi-baryon correlation function does not degrade exponentially faster that the signal-to-noise ratio in the single baryon correlation function. Instead,

$$
\frac{\sigma}{\bar{x}} \sim \frac{1}{\sqrt{\mathrm{N}}}\left(Z+Z^{\prime} \frac{A^{2}}{m_{\pi}^{3} L^{3}} e^{\left(M_{N}-\frac{3}{2} m_{\pi}\right) t}+\ldots\right)
$$

where $Z$ and $Z^{\prime}$ are $\mathscr{O}(1)$ ratios of overlap factors. Consequently, the signal-to-noise ratio starts degrading exponentially only after time-slice $t_{\text {noise }}$, which has parametric dependence

$$
t_{\text {noise }} \sim \frac{2}{2 M_{N}-3 m_{\pi}} \ln \left[\frac{m_{\pi}^{3} L^{3}}{A^{2}}\right] .
$$

It is important to note that $t_{\text {noise }}$ depends only logarithmically on the number of baryons.x

With these considerations in mind, it seems likely that the ground state energies of four- and five- baryon systems are resolvable at the current level of statistics. The impediment to such calculations is the computational requirements of the quark propagator contractions required to construct the appropriate correlation functions that grow factorially with the number of quarks involved. This computational constraint is the reason that the triton correlation function, which requires an order of magnitude more quark contractions, was evaluated with 1/30th of the statistical statistical precision of the " $\Xi^{0} \Xi^{0} n$ " correlation function (preliminary results for the triton effective mass are shown in Fig. ). Currently, progress is being made in constructing fast algorithms for such contractions.

\section{Conclusions}

The field of hadronic interactions and many body systems in lattice QCD has undergone tremendous progress over the last few years and is now a significant focus for a number of major lattice collaborations. Advances have been made in describing two hadron interactions and also in $n>2$ hadron systems. Perhaps the most far-reaching result of recent work is the recognition that the signal-to-noise issue that has kept lattice practitioners away from multi-baryon systems can be greatly reduced, opening the door for computations of the ground state energies of systems of four and more baryons. 


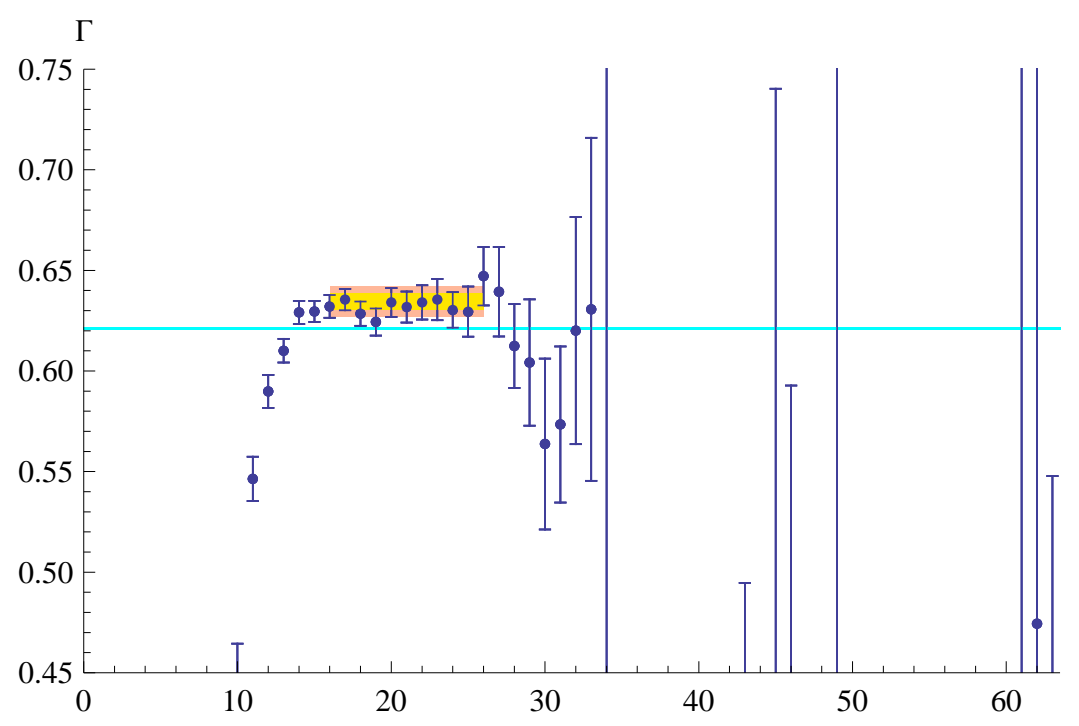

Figure 6: The generalized effective mass [22] for the $p p n$ correlation function (squares). The horizontal light band corresponds to three-times the nucleon mass.

\section{Acknowledgments}

This work has been supported by the US Department of Energy through Outstanding Junior Investigator grant DE-SC0001784 and by contract No. DE-AC05-06OR23177, under which Jefferson Science Associates, LLC operates Jefferson Laboratory.

\section{References}

[1] K. Huang and C. N. Yang, Phys. Rev. 105, 767 (1957).

[2] M. Lüscher, Commun. Math. Phys. 105, 153 (1986).

[3] S. R. Beane, W. Detmold, T. C. Luu, K. Orginos, M. J. Savage and A. Torok, Phys. Rev. Lett. 100, 082004 (2008) [arXiv:0710.1827 [hep-lat]].

[4] W. Detmold, M. J. Savage, A. Torok, S. R. Beane, T. C. Luu, K. Orginos and A. Parreno, Phys. Rev. D 78, 014507 (2008) [arXiv:0803.2728 [hep-lat]].

[5] W. Detmold, K. Orginos, M. J. Savage and A. Walker-Loud, Phys. Rev. D 78, 054514 (2008) [arXiv:0807.1856 [hep-lat]].

[6] S. R. Beane et al., Phys. Rev. D 80, 074501 (2009) [arXiv:0905.0466 [hep-lat]].

[7] S. R. Beane, P. F. Bedaque, A. Parreño and M. J. Savage, Phys. Lett. B 585, 106 (2004) [arXiv:hep-lat/0312004].

[8] C. J. D. Lin, G. Martinelli, C. T. Sachrajda and M. Testa, Nucl. Phys. B 619, 467 (2001) [arXiv:hep-lat/0104006].

[9] S. Aoki et al. [CP-PACS Collaboration], Phys. Rev. D 71, 094504 (2005) [arXiv:hep-lat/0503025].

[10] S. Aoki, T. Hatsuda and N. Ishii, arXiv:0909.5585 [hep-lat].

[11] W. Detmold, K. Orginos and M. J. Savage, Phys. Rev. D 76, 114503 (2007) [arXiv:hep-lat/0703009]. 
[12] S. R. Beane, K. Orginos and M. J. Savage, Int. J. Mod. Phys. E 17, 1157 (2008) [arXiv:0805.4629 [hep-lat]].

[13] S. R. Beane, PoS(LATTICE 2008)008, arXiv:0812.1236 [hep-lat].

[14] N. Ishii, these proceedings.

[15] K. Sasaki et al., these proceedings, arXiv:0911.0228

[16] X. Feng, K. Jansen and D. B. Renner, these proceedings, arXiv:0910.4871.

[17] K. J. Juge, et al., these proceedings, arXiv:0911.2044.

[18] X. Feng, K. Jansen and D. B. Renner, arXiv:0909.3255 [hep-lat].

[19] Q. Liu, these proceedings, arXiv:0910.2658.

[20] S. Prelovsek, et al., these proceedings, arXiv:0910.2749 [hep-lat].

[21] A. Torok et al., arXiv:0907.1913 [hep-lat].

[22] S. R. Beane et al., Phys. Rev. D 79, 114502 (2009) [arXiv:0903.2990 [hep-lat]].

[23] T. Inoue, these proceedings.

[24] NPLQCD Collaboration, in preparation.

[25] S. R. Beane, P. F. Bedaque, T. C. Luu, K. Orginos, E. Pallante, A. Parreño and M. J. Savage [NPLQCD Collaboration], Nucl. Phys. A 794, 62 (2007) [arXiv:hep-lat/0612026].

[26] H. Nemura, N. Ishii, S. Aoki and T. Hatsuda, Phys. Lett. B 673, 136 (2009) [arXiv:0806.1094 [nucl-th]].

[27] H. Nemura, N. Ishii, S. Aoki and T. Hatsuda [PACS-CS Collaboration], arXiv:0902.1251 [hep-lat].

[28] L. Liu, these proceedings.

[29] S. R. Beane, W. Detmold and M. J. Savage, Phys. Rev. D 76, 074507 (2007) [arXiv:0707.1670 [hep-lat]].

[30] S. Tan, arXiv:0709.2530 [cond-mat.stat-mech].

[31] W. Detmold and M. J. Savage, Phys. Rev. D 77, 057502 (2008) [arXiv:0801.0763 [hep-lat]].

[32] T. Luu, arXiv:0810.2331 [hep-lat].

[33] B. Smigielski and J. Wasem, Phys. Rev. D 79, 054506 (2009) [arXiv:0811.4392 [hep-lat]].

[34] D. B. Kaplan, Phys. Lett. B 288, 342 (1992) [arXiv:hep-lat/9206013].

[35] Y. Shamir, Nucl. Phys. B 406, 90 (1993) [arXiv:hep-lat/9303005].

[36] C. W. Bernard et al., Phys. Rev. D 64, 054506 (2001).

[37] S. R. Beane, T. C. Luu, K. Orginos, A. Parreno, M. J. Savage, A. Torok and A. Walker-Loud, Phys. Rev. D 77, 014505 (2008) [arXiv:0706.3026 [hep-lat]].

[38] D. T. Son and M. A. Stephanov, Phys. Rev. Lett. 86, 592 (2001) [arXiv:hep-ph/0005225].

[39] D. B. Kaplan and A. E. Nelson, preprint HUTP-86/A023; Phys. Lett. B 175 (1986) 57.

[40] T. Matsui and H. Satz, Phys. Lett. B 178, 416 (1986).

[41] W. Detmold and M. J. Savage, Phys. Rev. Lett. 102, 032004 (2009) [arXiv:0809.0892 [hep-lat]]. 
[42] R. G. Edwards, B. Joo and H. W. Lin, Phys. Rev. D 78, 054501 (2008) [arXiv:0803.3960 [hep-lat]].

[43] H. W. Lin et al. [Hadron Spectrum Collaboration], Phys. Rev. D 79, 034502 (2009) [arXiv:0810.3588 [hep-lat]].

[44] G. P. Lepage, 'The Analysis Of Algorithms For Lattice Field Theory,' Invited lectures given at TASI' 89 Summer School, Boulder, CO, Jun 4-30, 1989. Published in Boulder ASI 1989:97-120 (QCD161:T45:1989). 